\title{
Aspectos biométricos de frutos e de sementes de Tamarindus indica $\mathrm{L}$.
}

\section{Biometric aspects of fruit and seed of Tamarindus indica $L$.}

\author{
Laura Araujo Sanches ${ }^{1}$, Alcilene Batista de Camargo ${ }^{2}$, Juliana Garlet ${ }^{3}$
}

${ }^{1}$ Mestranda em Biodiversidade e Agroecossistemas Amazônicos, Universidade do Estado de Mato Grosso, Alta Floresta, (66) 9 97100393, laura_araujo_555@hotmail.com; ${ }^{2}$ Engenheira Florestal, Universidade do Estado de Mato Grosso, Alta Floresta, (66) 9 84577011, alcycamargo@ hotmail.com; ${ }^{3}$ Doutora em Engenharia Florestal, Professora da Faculdade de Ciências Biológicas e Agrárias,Universidade do Estado de Mato Grosso, Alta Floresta, (66) 999198748 , julianagarlet@unemat.br

\section{N O T A}

Recebido: $19 / 03 / 2018$

Aprovado: 07/12/2018

\section{Palavras-chave:}

Tamarindo

Biometria

Caracterização física

Key words:

Tamarind

Biometry

Physical characterization

\begin{abstract}
R E S U M O
Tamarindus indica L. é uma espécie arbórea muito apreciada para ornamentação, sendo considerada uma árvore multifuncional, uma vez que se pode utilizar a polpa, as sementes, as flores, as folhas, a madeira, a casca, seja para uso nutricional ou medicinal. Além disso, esta espécie apresenta em sua composição química aminoácidos, ácidos graxos, minerais, se destacando por apresentar excelentes qualidades nutricionais. Assim, o presente trabalho objetivou avaliar a biometria de frutos e sementes de Tamarindus indica L. Para isso, realizou-se a biometria utilizando uma amostra aleatória de 100 frutos e 100 sementes de Alta Floresta e da cidade de Juara, em Mato Grosso. Foram avaliadas a massa, o comprimento, a largura e a espessura de frutos e de sementes, e o número de sementes por fruto. Os frutos de Tamarindus indica apresentaram valores médios de comprimento, largura, espessura e massa de: 59,72; 22,80; 15, 29 mm e 7,78g respectivamente, com média de três sementes por fruto. Na análise biométrica das sementes observou-se médias de comprimento, largura, espessura, e massa de 14,$7 ; 10,15,3,85 \mathrm{~mm}$ e $1,12 \mathrm{~g}$ respectivamente.Os frutos e as sementes de Tamarindus indica apresentaram variabilidade em suas características biométricas, para as arvores avaliadas neste estudo. Os frutos apresentaram maior variação no comprimento, enquanto nas sementes foi observado maior variabilidade na espessura e na largura.
\end{abstract}

\begin{abstract}
A B S T R A C T
Tamarindus indica L. is a tree species very appreciated for ornamentation, being considered a multifunctional tree, since it can be used pulp, seeds, flowers, leaves, wood, bark, whether for nutritional or medicinal use. In addition, this species presents in its chemical composition amino acids, fatty acids, minerals, being outstanding for presenting excellent nutritional qualities. Thus, the present work aimed to characterize the biometry of fruits and seeds of Tamarindus indica L. For this, the biometry was performed using a random sample of 100 fruits and 100 seeds. Mass, length, width and thickness of fruits and seeds and number of seeds per fruit were evaluated. The fruits of Tamarindus indica presented average values of length, width, thickness and mass of: 59.72; 22.80; 15, $29 \mathrm{~mm}$ and $7.78 \mathrm{~g}$ respectively, with an average of three seeds per fruit. In the biometric analysis of the seeds we observed averages of length, width, thickness, and mass of $14.7 ; 10.15,3.85 \mathrm{~mm}$ and $1.12 \mathrm{~g}$ respectively. The fruits and seeds of Tamarindus indica showed variability in their biometric characteristics, for the trees evaluated in this study. The fruits presented greater variation in length, while in the seeds it was observed greater variability in thickness and width.
\end{abstract}

isso se deve a sua grande beleza e produção de sombra (PEREIRA et al., 2008).

É considerada uma árvore multifuncional,e quase todas as suas partes (polpa de frutas, sementes, flores, folhas, madeira, casca) podem ser utilizadas para alguma aplicação (KUMAR; BHATTACHARYA, 2008), seja nutricional ou medicinal. A polpa é utilizada como ingrediente em sendo uma espécie arbórea, que foi cultivada em praticamente todo o território brasileiro (FLORA BRASIL, 2018), tem origem da África e também é encontrada na Ásia, América do Sul e outras regiões tropicais. É uma espécie muito apreciada para ornamentação, mesmo apresentando crescimento lento,

\section{Revista Verde}

ISSN 1981-8203

Pombal, Paraíba, Brasil v. 14, n.1, jan.-mar, p.156-160, 2019

doi: $10.18378 /$ rvads.v14i1.5448 
condimentos e molhos e,também, é consumida como componente principal em sucos e certas bebidas, além disso, apresenta em sua composição química aminoácidos, ácidos graxos, minerais, se destacando por apresentar excelentes qualidades nutricionais $(\mathrm{Ca}, \mathrm{P}, \mathrm{Cu}, \mathrm{Mn}, \mathrm{Zn}$, elevado conteúdo de vitamina B) o aroma e sabor ácido-doce característico é devido a uma combinação de altos teores de ácido tartárico e redução de açúcares (CALUWÉ et al., 2010).

Pesquisas têm evidenciado a importância desta espécie, demonstrando que todas as partes da planta podem ser utilizadas, como na medicina popular, em que tem inúmeras aplicações terapêuticas em humanos, dentre essas pode-se citar o uso como: digestivo, calmante, laxante, expectorante e tônico sanguíneo (KOMUTARIN et al., 2004). Assim como também apresenta atividades farmacológicas, antiinflamatória e analgésica (SURALKAR et al., 2012).Essa importância ocorre devido aos seus múltiplos usos, e se faz necessário estudos sobre a tecnologia de suas sementes, buscando conhecer o comportamento da espécie, e das sementes nas diferentes regiões onde é cultivado. Além disso, são escassas estas informações no Mao Grosso, sendo este um trabalho inicial buscando conhecer o comportamento da espécie na região.

A biometria de frutos e sementes possibilita traçar uma estratégia para maximizar a uniformização de emergência das plântulas e obter mudas de padrões semelhantes ou de maior vigor através da classificação das sementes por tamanho e por peso (CARVALHO; NAKAGAWA, 2012). Além disso, expressa importantes informações sobre as diferentes características morfológicas entre indivíduos dentro de uma determinada área. Através de estudos biométricos é possível avaliar a variabilidade genética dentro e entre populações (GUSMÃO et al., 2006). A biometria dos frutos e sementes também é relevante para diferenciar a intensidade de variação das espécies relacionada a fatores ambientais, como as reações das populações quando estão estabelecidas em outro ambiente (RODRIGUES et al., 2006). Desta forma, o objetivo deste trabalho foi avaliar a biometria de frutos e sementes de Tamarindus indica L.

\section{MATERIAL E MÉTODOS}

O estudo foi conduzido no Laboratório de sementes da Universidade Estadual do Mato Grosso, no campus de Alta Floresta, Mato Grosso. As sementes de tamarindo utilizadas neste estudo foram coletadas de dez árvores matrizes com frutos maduros, provenientes de vários bairros distribuídos na cidade de Alta Floresta e na cidade de Juara, em Mato Grosso. As sementes foram despolpadas manualmente, lavadas em água corrente até a extração total do endocarpo, e colocadas para secar a sombra por 24 horas.

As determinações biométricas foram realizadas com auxílio de um paquímetro digital com precisão de $0,01 \mathrm{~mm}$, sendo utilizada uma amostra de 100 frutos e 100 sementes. As características biométricas avaliadas dos frutos e das sementes foram: comprimento determinado da base até o ápice, largura e espessura medidas na linha mediana dos frutos, e os valores máximo e mínimo de cada uma das dimensões. Além disso, determinou-se, também, o número de sementes por fruto, as massas individuais e de 100 frutos e 100 sementes. O número de sementes por fruto foi determinado pela contagem direta nos frutos.

Os dados obtidos foram submetidos à análise descritiva, obtendo-se as respectivas médias, desvio padrão e variância. Os dados da biometria dos frutos e sementes da espécie foram representados, graficamente, em histogramas de classes de frequência para cada variável, o número e intervalos de classe foi determinado, segundo Paiva (1982), através da fórmula de Sturges (Equação 1), sendo utilizada planilha eletrônica do Microsoft Office Excel.

$$
\mathrm{K}=1+3,33 \log \mathrm{n} \quad \text { Equação } 1
$$

Sendo: $\mathrm{K}=$ número de classes; $\mathrm{n}$ = número de dados avaliados.

\section{RESULTADOS E DISCUSSÃO}

A biometria dos frutos de Tamarindo foi determinada com base no comprimento, largura, espessura, conforme descrito na Tabela 1. Observa-se que os frutos de tamarindo apresentaram valores médios de comprimento, largura, espessura e massa de $59,72 \pm 13,01 ; 22,80 \pm 3,16,15,29 \pm 1,55$ $\mathrm{mm}$ e $7,78 \pm 2,89 \mathrm{~g}$ respectivamente. A massa de 100 frutos é de $778,41 \mathrm{~g}$.

Tabela 1. Dimensões de comprimento, espessura e largura de frutos de Tamarindus indica.

\begin{tabular}{|c|c|c|c|c|}
\hline Parâmetro & Comprimento (mm) & Largura $(\mathrm{mm})$ & Espessura (mm) & Massa dos frutos $(\mathrm{g})$ \\
\hline Média & $59,72 \pm 13,01$ & $22,80 \pm 3,16$ & $15,29 \pm 1,55$ & $7,78 \pm 2,89$ \\
\hline Mediana & 59,17 & 21,94 & 15,36 & 7,03 \\
\hline Variância & 169,34 & 10,00 & 2,40 & 8,33 \\
\hline $\mathrm{CV}(\%)$ & 21,79 & 13,86 & 10,13 & 37,07 \\
\hline
\end{tabular}

Nota-se, assim, que os frutos de tamarindo apresentam uma ampla variabilidade tanto no comprimento como na largura. Segundo Brito et al. (2014), a variabilidade nas dimensões de frutos pode ser influenciada pela abundância de nutrientes e água no período de formação do fruto. Além disso, Silva et al. (2017) também atribuem essa variação às condições edafoclimáticas de cada local de cultivo, sendo esse um dos fatores que pode estar influenciando na morfologia dos frutos.O comprimento, largura e espessura dos frutos, apresentaram baixo desvio padrão, no entanto, o maior valor foi observado para a característica comprimento, indicando que, possivelmente, esta seja uma variável de maior variabilidade em relação às demais. Quanto a variável massa dos frutos, nota-se um coeficiente de variação alto de $37,07 \%$ o que implica que houve uma maior variação da massa dos frutos.

O tamanho do fruto apresentou uma variação no comprimento, largura e espessura de 24,10 a 105,49; 17,59 a 31,96 e 9,63 a $18,74 \mathrm{~mm}$, respectivamente (Figura 1 A, B e C), e a média de massa dos frutos corresponde a 8,70 $\pm 9,61 \mathrm{~g}$. Observa-se que as classes mais representativas quanto ao número de frutos para as características de comprimento, largura e espessura foram respectivamente: 54,63 a $64,80 \mathrm{~mm}$ (30\%), 21,19 a 22,98 mm (33\%), 15,33 a 16,46 mm (31\%), e o maior número de frutos $(35 \%)$ apresentou valores entre 5,50 a 7,26g (Figura 1 D). 
Figura 1. Frequência (\%) para as características de comprimento (A), largura (B), espessura (C) e massa (D) de frutos de Tamarindus indica.

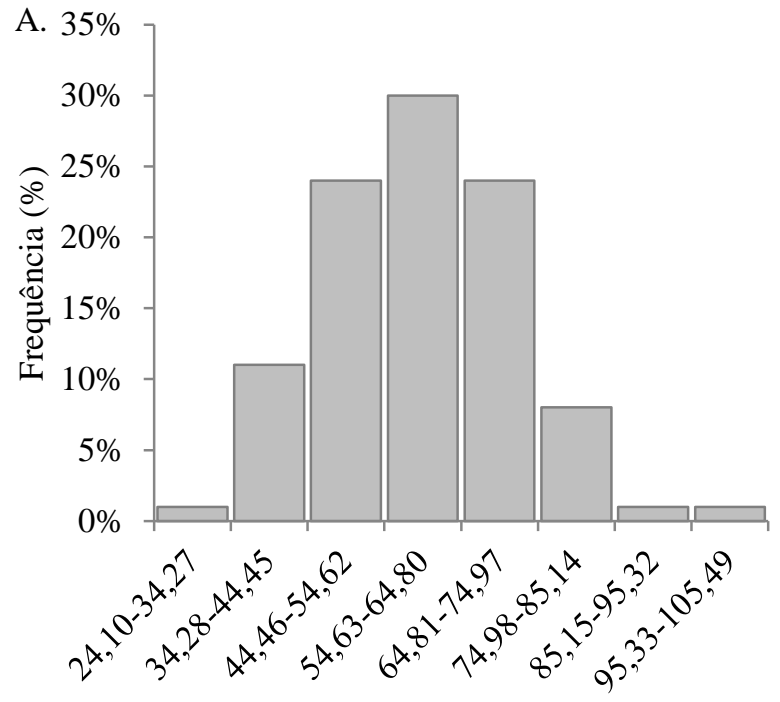

Comprimento $(\mathrm{mm})$

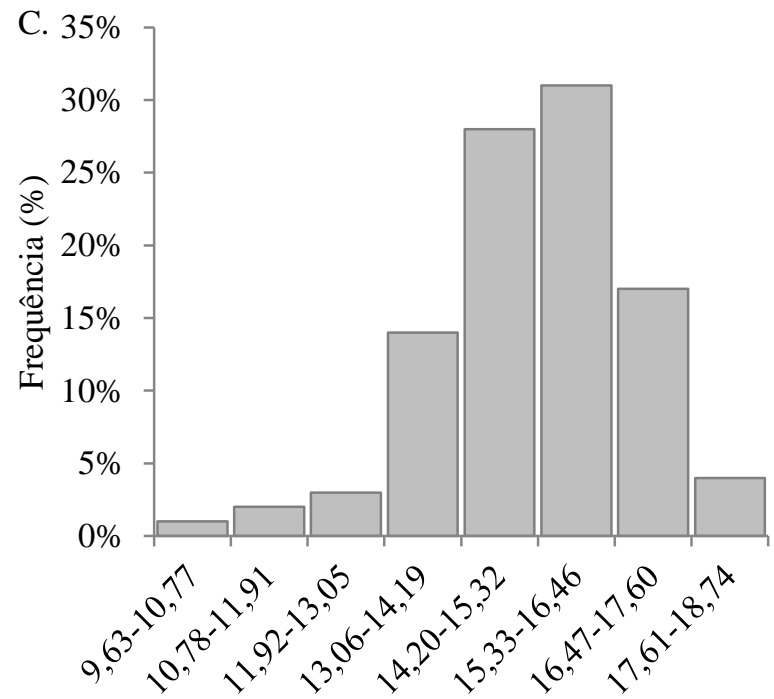

Espessura (mm)

A grande amplitude encontrada nas características do fruto de tamarindo pode ser atribuída ao fato de ser uma espécie arbórea tropical, Gusmão et al. (2006) mencionam que essas espécies, geralmente,apresentam grande variabilidade com relação ao tamanho dos frutos.

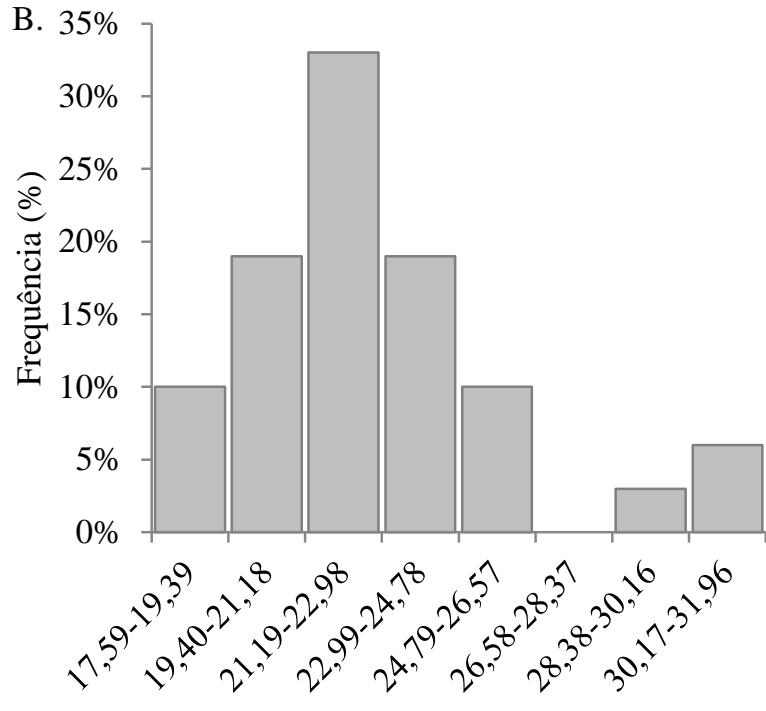

Largura (mm)

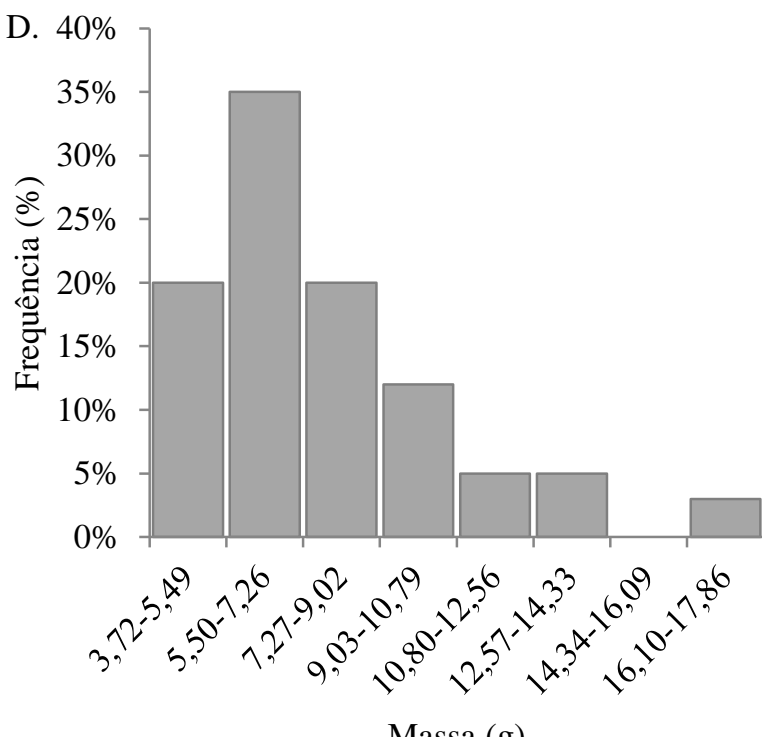

Massa (g)

Na Tabela 2 observa-se os dados de biometria das sementes de tamarindo. Com relação à biometria das sementes, a média de comprimento, largura, espessura, e massa de sementes foi de $14,7 \pm 2,36 \mathrm{~mm} ; 10,15 \pm 2,07 \mathrm{~mm}$, $3,85 \pm 0,83 \mathrm{~mm}$ e $1,12 \pm 0,16$ respectivamente. A massa de 100 sementes é de $112,45 \mathrm{~g}$.

Tabela 2. Dimensões de comprimento ( $\mathrm{mm})$, espessura ( $\mathrm{mm})$ e largura $(\mathrm{mm})$ de sementes de Tamarindus indica.

\begin{tabular}{lcccc}
\hline \multicolumn{1}{c}{ Parâmetro } & Comprimento $(\mathrm{mm})$ & Largura $(\mathrm{mm})$ & Espessura $(\mathrm{mm})$ & $\begin{array}{c}\text { Massa das } \\
\text { sementes }(\mathrm{g})\end{array}$ \\
\hline Média & $14,70 \pm 2,36$ & $10,15 \pm 2,07$ & $3,85 \pm 0,83$ & $1,12 \pm 0,16$ \\
Mediana & 14,00 & 10,00 & 4,00 & 1,12 \\
Variância & 5,59 & 4,27 & 0,69 & 0,02 \\
\hline CV $(\%)$ & 16,08 & 20,36 & 21,65 & 14,06 \\
\hline
\end{tabular}

As características avaliadas apresentaram baixo desvio padrão, entretanto, os maiores valores foram observados para espessura e largura, indicando que estas características apresentam maior variabilidade. Essa variação no tamanho das sementes dentro de uma mesma espécie pode estar associada com o ambiente, a falta de recursos necessários durante $\mathrm{o}$ desenvolvimento das sementes pode gerar diferentes padrões morfológicos, não apresentando uma uniformidade em seus tamanhos (SILVA et al., 2007). 
O comprimento, largura e espessura das sementes variaram de 9 a $21 \mathrm{~mm} ; 5$ a $14 \mathrm{~mm} 3$ a $6 \mathrm{~mm}$, respectivamente (Figura $2 \mathrm{~A}, \mathrm{~B}$ e C). A maior quantidade de sementes foi observada nos intervalos de classe para comprimento entre 12,01 a $13,50 \mathrm{~mm}$ e 15,01 a $16,50 \mathrm{~mm}$, ambos correspondendo a 29\% (58\%); largura de 9,51 a 10,63 $\mathrm{mm}(27 \%)$ e espessura de 3,00 a $3,38 \mathrm{~mm}(40 \%)$. A maioria dos frutos apresenta massa de sementes variando entre $0,92 \mathrm{e}$ $1,37 \mathrm{~g}$, ou seja, $85 \%$ das sementes estão compreendidas nas classes de massa com valores próximos da massa média das sementes, paralelamente foi observado que apenas $2 \%$ dos frutos apresentaram os menores valores de massa de sementes entre 0,57 e $0,80 \mathrm{~g}$ (Figura $2 \mathrm{D}$ ).

Figura 2. Frequência (\%) para as características de comprimento (A), largura (B), espessura (C) e massa (D) de sementes de Tamarindus indica.
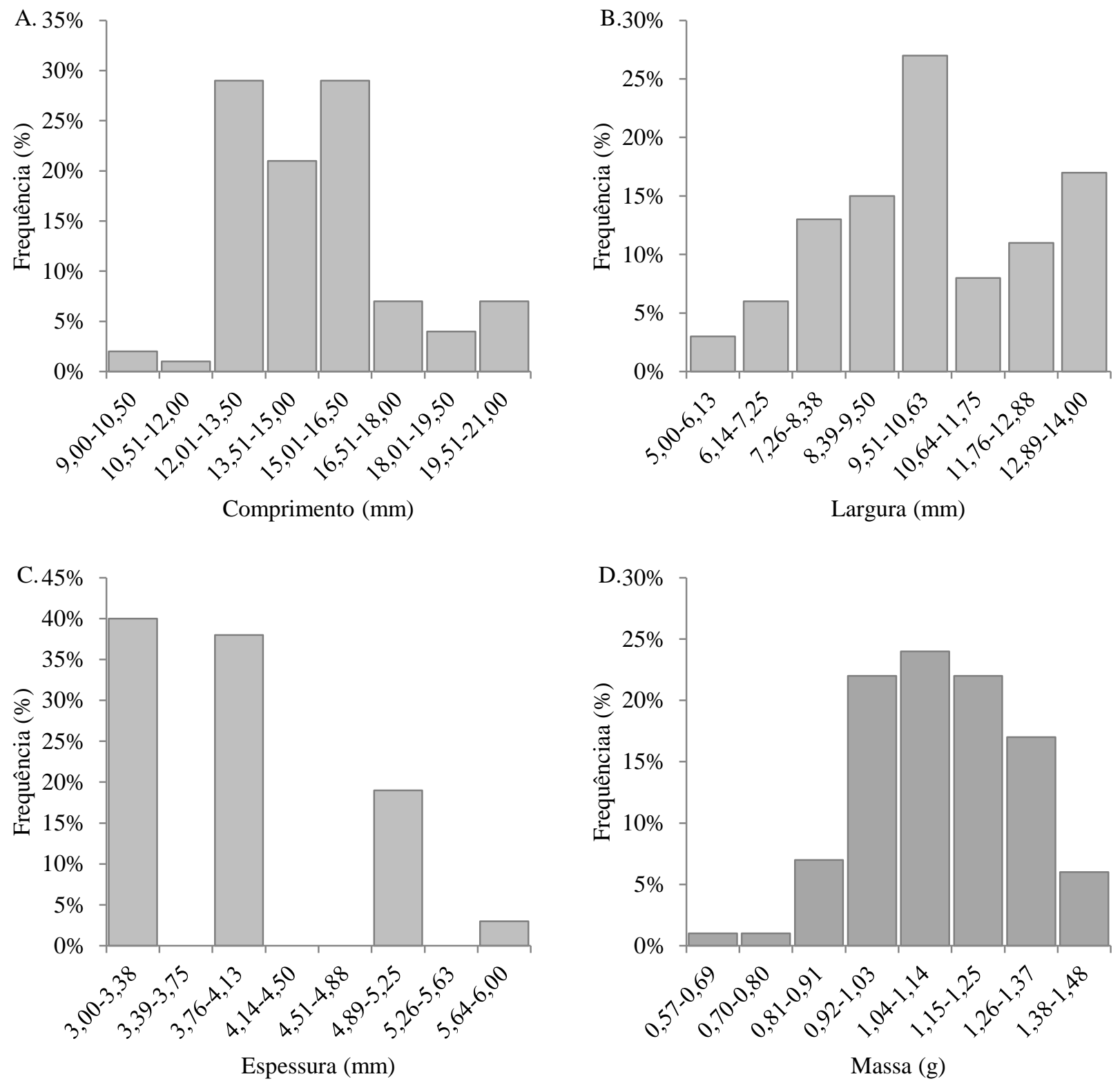

Embora as sementes sejam formadas pelo embrião, tecidos de reserva e envoltório, diversos fatores podem promover um desenvolvimento diferenciado dos componentes da semente, ocasionando variações, seja entre espécies, seja até dentro da mesma espécie, e esta variação é vista através da cor, da forma e do tamanho (ABUD et al., 2010). Geralmente, as sementes maiores podem produzir mudas maiores, essas podem superar as mudas ao seu redor (KUMAR et al., 2016). Segundo Macedo et al. (2009), a variação das dimensões das sementes são menores, quando comparadas com o fruto.

A massa de sementes e o teor de açúcar indicam a reserva de energia para a síntese de outras moléculas

orgânicas para começar o primeiro estágio da vida até que as mudas se tornem autotróficas (QUERO et al., 2007; DEKKERS; SMEEKENS, 2007; SORIANO et al., 2013). Além disso, a massa é considerada uma característica que pode ser utilizada como indicadora da qualidade fisiológica das sementes (COSTA et al., 2006).

Pereira et al. (2008) verificaram que as sementes com maior massa proporcionaram melhores mudas, considerando o tamanho da semente como um determinante na qualidade da muda desta espécie, sendo que sementes grandes e muito grandes produziram plantas de maior diâmetro de coleto, maior massa seca do sistema radicular e da parte aérea. Os 
mesmos ressaltaram a importância da seleção e da classificação das sementes para obtenção de mudas vigorosas e uniformes, sendo que sementes de massa e de dimensões menores apresentam maiores porcentagem de emergência, enquanto que sementes de maior tamanho proporcionam plantas de tamarindo de qualidade superior.

$\mathrm{O}$ número de sementes por fruto variou de um a oito, sendo verificada a média de $3 \pm 1,26$ sementes por fruto. De acordo com Nascimento et al. (2011), a variação do número de sementes por fruto está relacionada com o processo de polinização, sendo que maior será o número de sementes quanto maior for o número de óvulos fertilizados. Esses fatores são importantes quando se busca maior produção de polpa.

\section{CONCLUSÃO}

Os frutos e as sementes de Tamarindus indica tem variabilidade em suas características biométricas, com valores médios de comprimento, largura, espessura e massa de frutos de: 59,$72 ; 22,80 ; 15,29 \mathrm{~mm}$ e $7,78 \mathrm{~g}$ respectivamente. Com relação ás sementes, a média de comprimento, largura, espessura, e massa foi de 14,$7 ; 10,15,3,85 \mathrm{~mm}$ e $1,12 \mathrm{~g}$ respectivamente. Os frutos apresentam maior variação no comprimento, enquanto as sementes tem maior variabilidade na espessura e na largura.

\section{REFERÊNCIAS}

ABUD, F. H.; GONÇALVES, N. R.; REIS, R. G. E.; GALLÃO, M. I.; INNECCO, R. Morfologia de sementes e plântulas de cártamos. Revista Ciência Agronômica, Fortaleza, v. 41, n. 2, p. 259-265, 2010.

BRITO, S. F.; SOUSA, J. E. S.; SILVA, J. A.; BEZERRA, A. M. E. Morfometria de frutos e sementes e desenvolvimento pós-seminal de Acnistus arborescens. Revista Ciências Agrárias, v. 57, n. 4, p. 422-428, 2014.

CALUWE, E.; HALAMOVA, K.; DAMME, P. V. Tamarindus indica L. - A reviewoftraditional uses, phytochemistry and pharmacology. Afrika Focus, v. 23, n. 1, p. 53-83, 2010.

CARVALHO, N. M., NAKAGAWA, J. Sementes: ciência, tecnologia e produção. 5.ed. Jaboticabal: FUNEP. 590p,

2012.

COSTA, R. S.; OLIVEIRA, I. V. M.; MÔRO, F. V.; MARTINS, A. B. G. Aspectos morfológicos e influência do tamanho da semente na germinação do jambo-vermelho. Revista Brasileira de Fruticultura, v. 28, n. 1, p. 117-120, 2006.

DEKKERS, B.J.W., SMEEKENS, S.C.M. Sugar and abscisic acid regulation of germination and transition to seedling growth. In: BRADFORD, K., NONOGAKI, H. (Eds.), Seed Development, Dormancy and Germination. Blackwell Publishing, Oxford, UK, p. 305-327, 2007.

GUSMÃO, E.; VIEIRA, F. A.; FONSECA, E. M. Biometria de frutos e endocarpos de murici (Byrsonima verbascifolia Rich. Ex A. Juss.). Cerne, Lavras, v. 12, n. 1, p.84-91, 2006.

JARDIM BOTÂNICO DO RIO DE JANEIRO. Disponível em:

<http://floradobrasil.jbrj.gov.br/reflora/floradobrasil/FB23201

$>$. Acesso em: 11 Jan. 2018.
KOMUTARIN, T.; AZADI, S.; BUTTERWORTH, L.; KEIL, D.; CHITSOMBOON, B.; SUTTAJIT, M.; MEADE, B. J. Extract of the seed coat of Tamarindus indica inhibits nitric oxide production by murine macrophages in vitro and in vivo. Food and Chemical Toxicology, v. 42, n. 4, p. 649-658, 2004.

KUMAR, C. S.; BHATTACHARYA, S. Tamarind Seed: Properties, Processing and Utilization. Critical Reviews in Food Science and Nutrition, v. 48, n. 1, p. 1-20, 2008.

KUMAR, R., SHAMET, G.S., ALAM, N. M., JANA, C. Influence of growing medium and seed size on germination and seedling growth of Pinus gerardiana wall. Compost Science \& Utilization, v. 24, n. 2, p. 98-104, 2016.

MACEDO, M. C.; SCALON, S. P. Q.; SARI, A. P.; SCALON FILHO, ROSA, H. Y. B. C. J.; ROBAINA, A. D. Biometria de frutos e sementes e germinação de Magonia pubescens ST.Hil (Sapindaceae). Revista Brasileira de Sementes, v. 31, n. 2, p.202-211, 2009.

NASCIMENTO, W. N.; LIMA, G. P.; CARMONA, R. Influência da quantidade de pólen na produção e qualidade de sementes híbridas de abóbora. Horticultura brasileira, Brasília, v. 29, n. 1, p. 29-25, 2011.

PAIVA, A. F. Estatística. Belo Horizonte: UFMG, 1982. 475p.

PEREIRA, P. C.; FREITAS, R. S.; MELO, B.; FRANZÃO, A. A.; PEREIRA, A. P.; SANTANA, J. G.; LUZ, J. M. Q.; MARTINS, M. Influência do tamanho de sementes na qualidade de mudas de tamarindeiro. Bioscience Journal, v. 24, n. 4, p. 73-79, 2008.

QUERO, J. L.; VILLAR, R.; MARAN, T.; ZAMORA Ó, N. R.; POORTER, L. Seed-mass effects in four Mediterranean Quercus species (Fagaceae) growing in contrasting light environment. American Journal of Botany, v. 94, n. 11, p. 1795-1803, 2007.

RODRIGUES, A. C. C.; OSUNA, J. T. A.; OLIVEIRA, S. R.; QUEIROZ, D.; RIOS, P. S. Biometria de frutos e sementes e grau de umidade de sementes de angico (Anadenanthera colubrina (Vell.) Brenan Var. cebil (Griseb.) Altschul) procedentes de duas áreas distintas. Revista Científica Eletrônica de Engenharia Florestal, v.4, n.8, p.1-15, 2006.

SILVA, D. D. A.; MACHADO, C. G.; CRUZ , S. C. S.; VESPUCCI, I. L.; ARAUJO, Y. J. D. Temperatura e substrato para o teste de germinação de sementes de tamarindo. Revista Espacios, v. 38, n. 14, p. 4-14, 2017.

SILVA, P. S. L.; SILVA, K. M. B.; LÔBO, R. N. B.; SILVA, P. I. B. Growth of seven perennial plant species adapted to the Brazilian Semi-Arid. Acta Botanica Brasilica, v. 21, n. 4, p. 935- 941, 2007.

SORIANO, D.; HUANTE, P.; GAMBOA-DE BUEN, A.; OROZCO-SEGOVIA, A. Seed reserve translocation and early seedling growth of eight tree species in a tropical deciduous forest in Mexico. Plant Ecolgy, v. 214, n. 11, p. 1361-1375, 2013.

SURALKAR, A. A.; RODGE, K. N.; KAMBLE, R. D.; MASKE, K. S. Evaluation of anti-inflammatory and analgesic activities of Tamarindus indica seeds. International Journal of Pharmaceutical Sciences and Drug Research, v. 4, n. 3, p. 213-217, 2012. 